\title{
CONTROL OF VORTEX SHEDDING AROUND A CIRCULAR CYLINDER USING BUBBLE TABS IN THE LAMINAR FLOW REGIME
}

\author{
E. O. Atofarati ${ }^{1}$, A. O. Muritala ${ }^{2}$, B. O. Malomo ${ }^{3, *}$ and S. A. Adio ${ }^{4}$ \\ $\mathbf{1 , 2 , 3 , 4}$, Department of MeChanical Engineering, Obafemi Awolowo University, Ile-Ife, OSUn State, \\ NIGERIA \\ Email addresses: ${ }^{1}$ atofaratiemmanuel@yahoo.com, ${ }^{2}$ muriadam@oauife.edu.ng, \\ 3 bobmalom@oauife.edu.ng; ${ }^{4}$ adiosa@oauife.edu.ng
}

\begin{abstract}
Vortex induced vibration (VIV) is the major cause of several catastrophic disasters due to fatigue failures induced by drag and lift forces in aerodynamic systems. This study investigates the control of VIV phenomenon through passive bubble tab(s) having a small diameter (d) relative to the main circular cylinder (D) in a two-dimensional (2-D) flow domain. Using ANSYS Fluent computational software, flow analysis was conducted at a Reynolds number (Re) of 80 for various bubble tab configurations at different spacing ratios $(x / D)$ and diameter ratios $(d / D)$. The drag coefficient, the velocity and pressure contours, along with the flow streamlines in each case were studied. The results indicated the optimized tab(s) positions for different spacing ratios, diameter ratios, and configurations. The study effectively established that passive bubble tabs can potentially control VIV associated with flows around a circular cylinder.
\end{abstract}

Keywords: Vortex Shedding; Drag Coefficient; Circular Cylinder; Bubble Tab(S); Spacing Ratio; Diameter Ratio.

\section{INTRODUCTION}

Control of fluid flow around a circular cylinder is a highly fascinating study due to its vast technological applications and its capability to address certain challenging aspects in aerodynamics. Diverse relevant engineering structures such as an aircraft, submarine, skyscraper (buildings), antennas, masts, towers, power lines, and bridges have their basic geometries modelled from a circular cylinder. Around circular cylinders, it is well-known that the phenomenon of vortex shedding occurs when vortices are generated downstream, at a Reynolds number between $(44 \leq \operatorname{Re} \leq 47)[1]$. Hence the flow pattern can be subject to control through the Reynolds number, which depends on the kinematic viscosity $\left(\frac{\mu}{\rho}\right)$ of the moving fluid, the free-stream velocity $(u)$, and the cylinder diameter $(d)$.

Several engineering structures, such as automobiles, chimneys, and aircraft have been subjected to poor performance and high cost (fuel) of operation due to the dearth of research findings on the control of vortex shedding in aerodynamic designs. Vortex shedding has posed various defects such as drag enhancement, lift fluctuation, noise pollution, and vibration induction. For instance, Holmes [2], had reported the collapse of the tall power plant chimneys designed by the masonry construction. The author illustrated that the failure occurred due to the overload caused by vortex shedding generated around it during a windstorm as fatigue stress was imposed on the structure at resonance, with the natural frequency of the structure attaining the vortex shedding frequency of the (air) flow. Experimental studies have shown that vortices formed around cylinders can be disrupted by placing helical strakes around its boundary layer [3]. This work revealed that helical strakes are often used to control vortex shedding around tall chimneys used in industries. However, the proximity of other structures reduces the effectiveness of helical strakes [4]. Due to the universal concept of flow around a bluff body, the effect of the various

${ }^{*}$ Corresponding author, tel: $+234-806-473-6088$ 
controlling objects around the boundary layer may alter their aerodynamic behavior (i.e. drag and lift force, vortex frequency). The controlling object may be a helical strake as stated earlier, flexible plate, rigid plate, a small rod, multiple small rods, undulating plate, or bubble tabs. It could be arranged either in a side-by-side pattern in relation to the main cylinder (i.e. anti-phase) [5] or in a tandem arrangement (i.e. in-phase) $[6,7]$.

Controls of vortex shedding around a circular cylinder have been an innovative solution to the engineering problem of VIV. VIV control problems have been approached experimentally, theoretically, and numerically in a systematic manner for proper understanding, predictions, and further application [5]. Many interesting control phenomena may occur when two or more bodies are placed beside one another in order to form groups. This grouping effect of bodies is a vital subject of fundamental research in fluid mechanics. Two circular cylinders of equal diameters arranged side-by-side is a simple case of such a group. Other types of such group is a cylinder and small $\operatorname{rod}(\mathrm{s})$, a cylinder and flexible plate, a cylinder and rigid plate, the cylinder and an undulating plate, and a cylinder and bubble tabs. The experimental observation of flow past two cylinders of the same size arranged side-by-side in a laminar flow regime has been conducted [8] and it was revealed [9] that the vortex-shedding phenomenon occurs either in-phase (i.e. when on a straight line) flow or in anti-phase (i.e. not in a straight line). The authors concluded that the position and the gaps between the cylinders had a great effect on the rate of periodic change of the vortex shedding. The simulation report of [10], suggested some periodic changes of the vortex shedding around the cylinder, leading to the conclusion that the vortex shedding generates a periodic variation of the force component i.e., drag and lift forces. The forces in the in-line and cross-flow directions are the 'drag and the lift' forces respectively. It was further stated that in the flow downstream the pressure distribution generates the drag force. Further to this, the measurement of the drag and lift forces for two circular cylinders placed in tandem was experimentally carried out by [4]. In the same vein a correlation between the drag and lift forces to the measured values of the pressure distribution around the cylinder has been achieved [11]. Also, the pressure distributions and lift/drag coefficients for flow around four cylinders with varied spacing ratio from 1.26 to 5.8 have been characterized [12].
However, it was observed by [5] that the vortex shedding of a cylinder could be eliminated while the drag/lift reduction may attain $85 \%$ as compared to the scenario without a traveling wave. Numerically, the authors investigated the control of flows around a stationary circular cylinder in a laminar regime using $\operatorname{Re}=100$, where an undulatory or flexible plate was used as the control object while varying the frequency and amplitude of the plate. Some more effective drag reduction and flow patterns were observed when the results were compared with previous numerical control of flows around a circular cylinder where the rigid plate and flexible plate were used as control objects. The experimental investigation by [7], elicited the mechanism behind the control of VIV using multiple small rods; it was observed that some degree of adequate minimization of VIV responses were achieved with the aid of these controls.

Nonetheless, due to the complications in instrumentation needed in measuring the fluid forces and recording the flow pattern images. The experimental approach to the study of VIV suppression using multiple control rods remains unclear. This is where this research draws its motivation; objectively, the emphasis is to study the control of vortex-induced vibration around a circular cylinder numerically using bubble tabs as the control objects in a laminar flow regime through the application of the concept of Direct Numerical Simulation (DNS) to achieve a reduction in the effect of the drag force.

\section{METHODOLOGY}

\subsection{Governing Equations}

A simplified form of the Navier-Stokes equations for incompressible Newtonian fluids and the continuity equation are given as follows. The fluid of density $\rho$, is considered to flow past the circular cylinder of diameter $\mathrm{D}$, with a stream-wise velocity $\mathrm{U}(\mathrm{u}, \mathrm{v})$ :

$$
\begin{aligned}
& \frac{\partial \mathrm{u}}{\partial \mathrm{x}}+\frac{\partial v}{\partial y}=0 \\
& \rho X-\frac{\partial p}{\partial x}+\mu\left(\frac{\partial^{2} u}{\partial x^{2}}+\frac{\partial^{2} u}{\partial y^{2}}\right)=\rho \frac{D u}{D t} \\
& \rho Y-\frac{\partial p}{\partial y}+\mu\left(\frac{\partial^{2} v}{\partial x^{2}}+\frac{\partial^{2} v}{\partial y^{2}}\right)=\rho \frac{D v}{D t}
\end{aligned}
$$

\subsection{Numerical Approach}

Posdziech and Grundmann [13] studied the effect of domain size when the time step is set as $\Delta t=0.005$ 
at Reynolds number ranging from $50 \leq R e \leq 250$. The minimum domain size required was dependent on the Reynolds number, as well as the boundary conditions (the no-slip boundary condition and shear boundary condition). The flow domain in this research is a rectangular domain defined by the diameter ' $D$ ' of the main cylinder and with a dimension of $30 \mathrm{D} \times 15 \mathrm{D}$; in the Cartesian Coordinates $\mathrm{X}$ and $\mathrm{Y}$ indicating the stream-wise and cross-stream directions respectively. Figures 1(a) and (b) represent the computational domain of an isolated main cylinder (c) and is a generated mesh/grid for a twin bubble tab configuration respectively.

The bubble tab configurations considered are the single bubble tab and the twin bubble tab with the bubble tab being specified at the stagnation point axis, adjacent to the separation point, and at an inclination angles of $30^{\circ}$ and $15^{\circ}$ from the x-axis.

At the respective tab locations defined above, the spacing ratio $(x / D)$ and the diameter ratio $(d / D)$ was varied for proper observation of the flow behavior and the optimized position was determined thereafter as shown in Figure 1 (b). The results presented indicate the drag coefficients at different positions of the bubble tabs, for the various number of bubble tabs, spacing ratios, diameter ratios, and Reynolds numbers respectively; with regard to the most efficient number of bubble tabs that indicate an effective drag reduction.

\section{RESULTS AND DISCUSSION}

\subsection{Authenticity of Simulation Physics:}

Through parallel comparison of the simulation results with simulations and experiments of previous literature, the results of this study were validated and the physics was established by verifying the convergence of results and mesh independence of the circular cylinder (isolated). The validating results as compared with previous research results are shown in Table 1.

Table 1 Verification result at different Reynolds number

\begin{tabular}{ccccc}
\hline $\begin{array}{c}\text { Reynolds } \\
\text { number }\end{array}$ & $\begin{array}{c}\text { Cd } \\
\text { present } \\
\text { study }\end{array}$ & $\begin{array}{c}\text { Cd } \\
\text { previous } \\
\text { literature study }\end{array}$ & $\begin{array}{c}\text { Strouhal } \\
\text { Number }\end{array}$ & $\begin{array}{c}\text { Strouhal Number } \\
\text { previous literature } \\
\text { study }\end{array}$ \\
\hline 40 & 1.7162 & $1.54[14], 1.73[15]$ & - & - \\
50 & 1.5376 & $1.54[14], 1.52[6]$ & - & - \\
80 & 1.4034 & $1.40[15], 1.46[16]$ & 0.1578 & $0.158[6]$ \\
100 & 1.3814 & $1.39[14], 1.45[15]$ & 0.1490 & $0.168[17]$ \\
150 & 1.3659 & $1.36[14], 1.37[15]$ & 0.1495 & - \\
200 & 1.3725 & $1.33-1.38[18], 1.32$ & 0.1592 & $0.192[19]$ \\
& & {$[19]$} & & \\
\hline
\end{tabular}

\subsection{Flow Behavior around Main Cylinder (Isolated)}

The purpose of this study is to verify the precision of the ANSYS Fluent physics formulations used in this research in comparisons to previous experiments and research results.

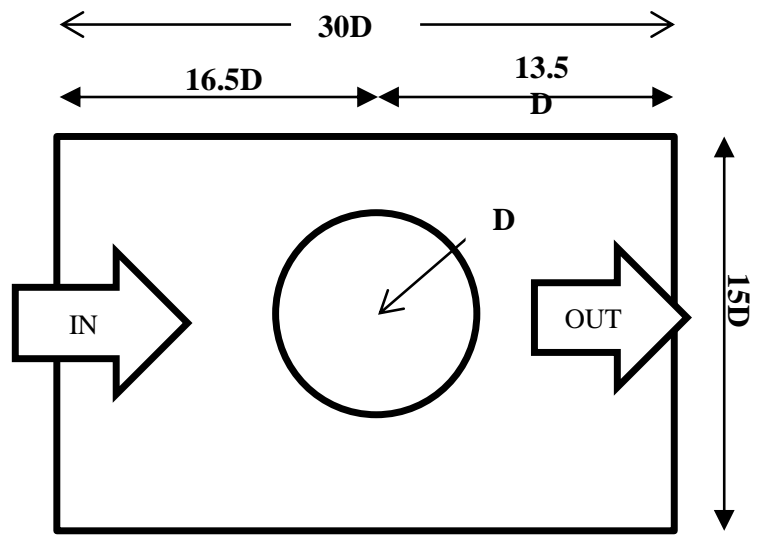

(a)

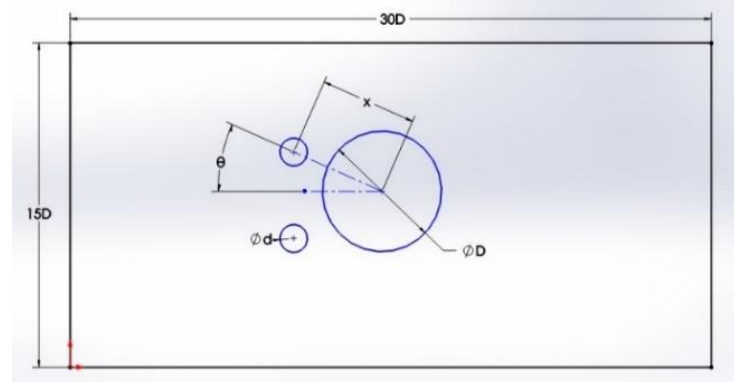

(b)

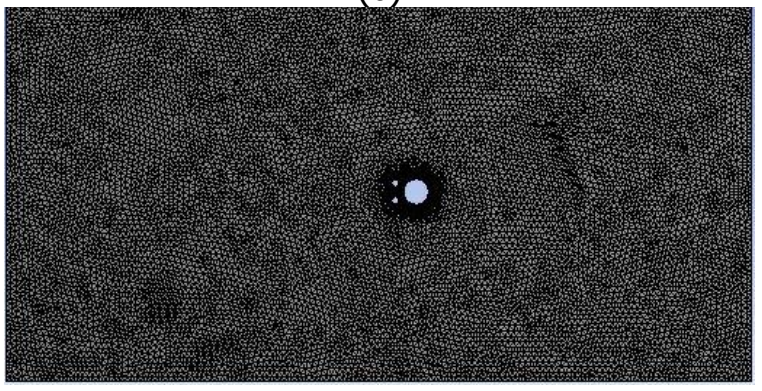

(c)

Figure 1: (a) Computational Domain (b) Domain for twin tab cylinder (c) Grid for the Twin-bubble tabs. 
This was achieved by comparing the Strouhal number and Drag coefficient for the flow around the single (tab-less) cylinder at different Reynolds number $(40,50,80,100,150$, and 200). The results, as presented in Table 1 validate the mathematical model solved by the Fluent solver. Having considered the result of the drag coefficient and the Strouhal number for the flow around an isolated main cylinder, these results were compared with the outcome of previous simulation and experimental works and were found to be in close agreement.

\subsection{Optimization of Bubble Position and Results}

To achieve an accurate control of the flow around the circular cylinder, the effect of the single and twin bubble-tab was studied.

\subsubsection{The Single Bubble Tab}

In the 2D model for a single bubble at $\operatorname{Re}=80$, it was observed that the drag coefficient which is an essential factor to be considered in the control of vortex shedding was found to be reduced by $55.85 \%$ when compared with that of the main cylinder. These bubble tab diameter ratios, angular position, and spacing ratios were varied until an optimized position was found. An optimized drag coefficient of 0.6269 was obtained when the bubble tab diameter ratio (d/D) was 0.5 and spacing ratio ( $\mathrm{x} / \mathrm{D}$ ) was 1.2 , along the stagnation point axis of the main cylinder in the flow upstream as shown in Figures 2 (a) and (b).

In Figure $2(a)$, it was observed from the dragcoefficient versus flow-time plot that the coefficient of drag for the tabbed cylinder indicated a significant reduction compared to that of the main cylinder only, as the flow time progressed. A steady and stabilized drag coefficient value of 1.1 was recorded after a flow time of $1.7 \mathrm{~s}$. Figure 2 (b) shows the coefficient of drag for different tab positions (design point). The plot reveals the 'stagnation point' position as the optimized tab positioning in comparison with the other positions of the bubble tab on the main cylinder (tab-less).

Effect of Spacing Ratio: The drag coefficient tends to decrease proportionally as the spacing ratio of the bubble tab increased. This inverse relation justifies the reason why helical stakes are placed at the boundary layer of a chimney and not too far from it. The relationship between the spacing ratio and the drag coefficient at diameter ratio $(d / D)=0.2$ is shown in Figure 3 (a).

Effect of Diameter Ratio: The diameter ratio of the bubble tab was varied at a spacing ratio $(x / D)=$ 0.9 and the drag coefficient was observed to decrease when positioned at the stagnation axis, i.e., $15^{0}$ from the stagnation axis, and $30^{\circ}$ from the stagnation point respectively, except at the position of the separation point where it increases. Figure 3 (b) presents the relations between the coefficient of drag and the diameter ratio at a fixed spacing ratio. It was also observed from the results that the flow separation was delayed due to the interactions between the vortices generated by the bubble tab and those generated by the main cylinder, thus reducing the quick formation of the Karman street; a scenario indicating the occurrence of significant reduction of vortex shedding.

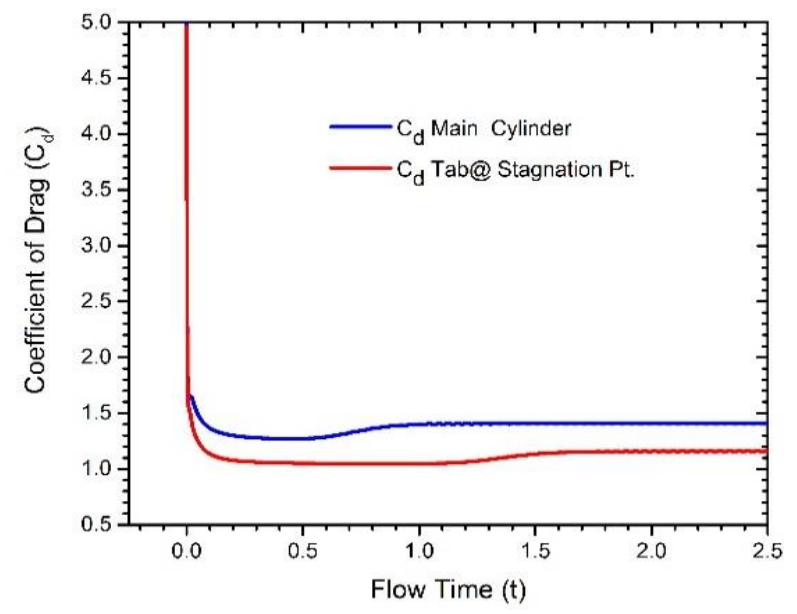

(a)

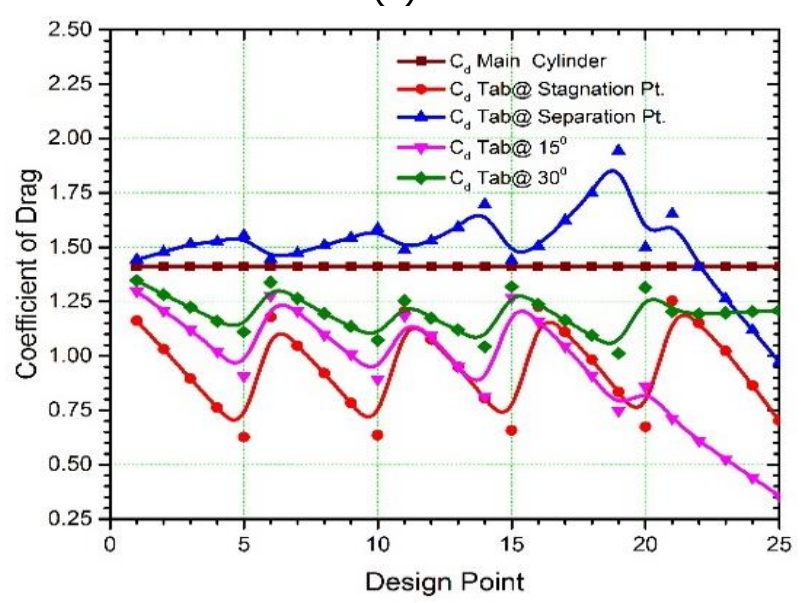

(b)

Figure 2: (a) Comparison of the Cd plot of main cylinder and position $(x / D=1.2, d / D=0.5)$

Determination of Optimized position of bubble tab (Single tab) 
Figure 4 shows the vorticity contour at $\operatorname{Re}=80$, where the streamlines of the flow generated showed a delay in flow-time and distortion in the pressure distribution formed downstream instead of the uniform pressure distribution formed in the isolated

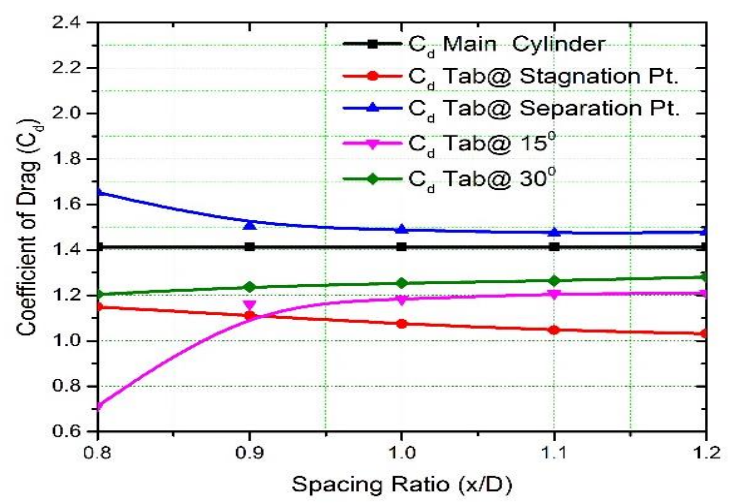

(a) main cylinder. Consequently, the lower vortex is eradicated leaving the upper vortex with a transformed pressure distribution as shown in Figure 5 , whereas in Figure 6 the pressure contour at the optimized position is significant.

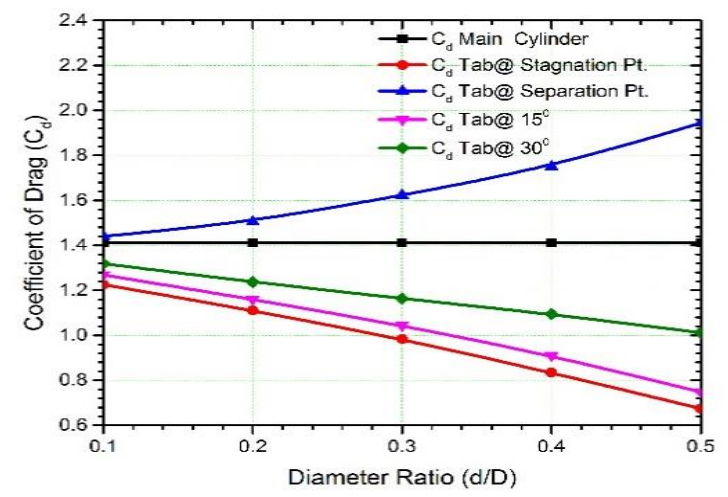

(b)

Figure 3 (a): Effect of spacing ratio on drag coefficient (at constant diameter ratio) (b) Effect of diameter ratio on drag coefficient (at constant spacing ratio)

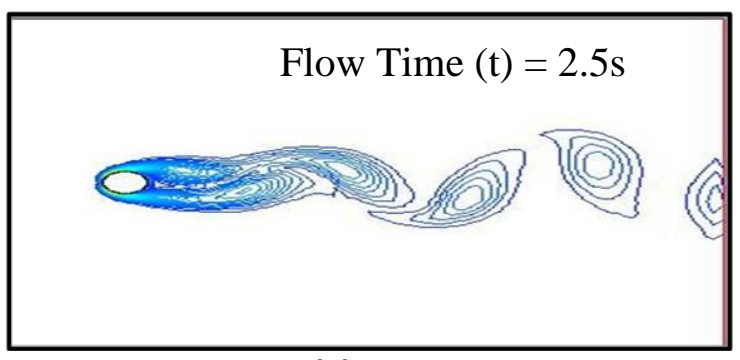

(a)

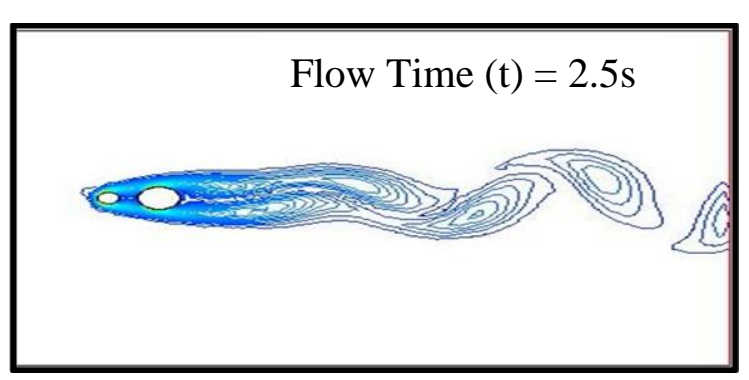

(b)

Figure 4: Vorticity contour for the main cylinder only and optimized single bubble tab (a) Main cylinder only (b) Main cylinder with a single tab at the optimized position

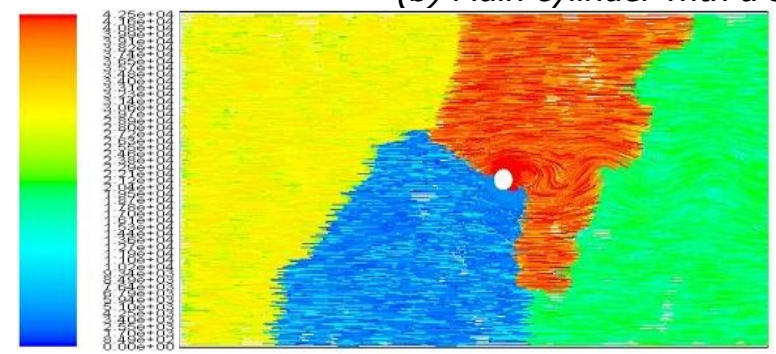

(a)

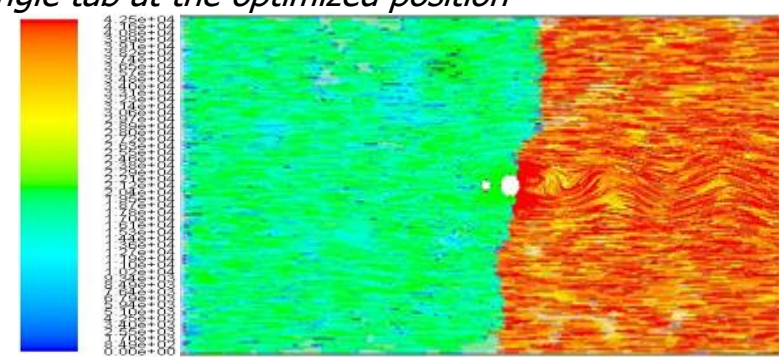

(b)

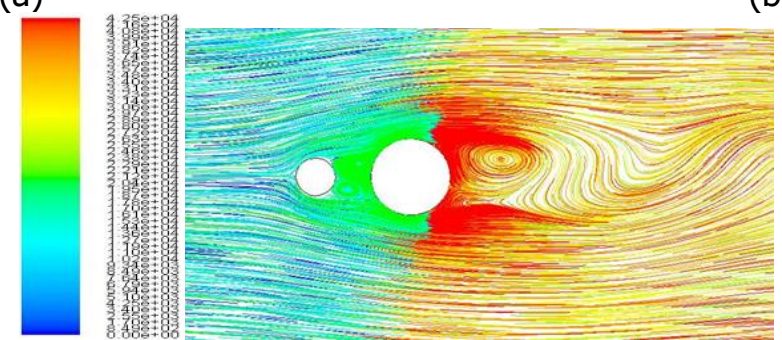

(c)

Figure 5: Streamline of flow for isolated main cylinder and Optimized single tab. (a) Isolated main cylinder (b) single tab at optimized position (c) zoomed ' $b$ ' 

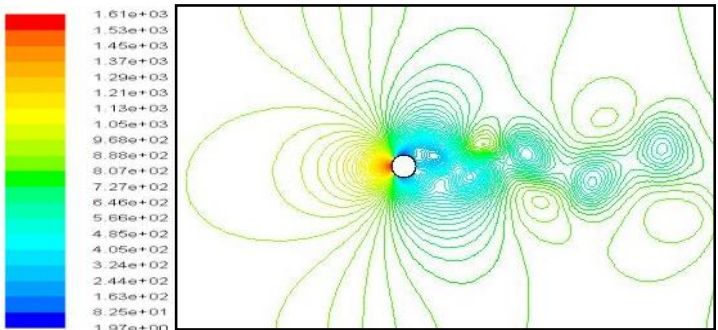

(a)
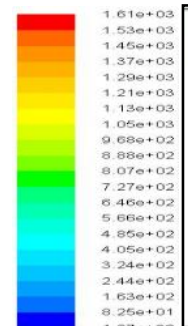

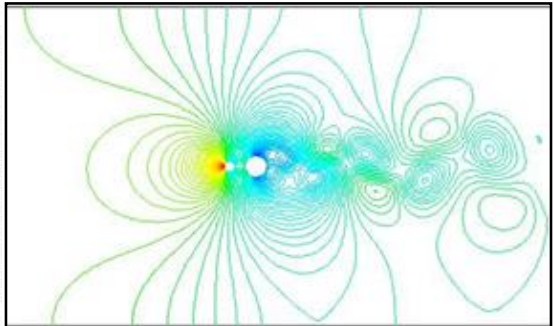

(b)

Figure 6: Pressure contour at the optimized position, (a) Main cylinder only (b) Main cylinder with a single tab at the optimized position

Effect of Reynolds Number at Optimized Position: Further investigation on the Reynolds number effect on drag coefficient at the optimized position established that an inverse relationship exists between the drag coefficient and Reynolds number for the main cylinder (tab-less) while the optimized single cylinder is directly related. In Figure 7 that drag coefficient was shown to have a tendency to decrease mildly as the Reynolds number increases for the main cylinder (tab-less) while for the optimized single cylinder, it considerably increases as the Reynolds number increases, even though the drag coefficient value remained stable up to $\mathrm{Re}=$ 100. Nonetheless, the value was observed to increase spontaneously from about $\operatorname{Re}=120$.

\subsubsection{The Twin Bubble Tab}

For the twin bubble tab, two equal bubble tabs were placed in the flow upstream at $\mathrm{Re}=80$ considering different positions to determine the optimized position for which a drastic drag reduction would be achieved to suppress the vortex shedding around the main cylinder. It follows that an optimized position was realized at a spacing ratio (x/D) of 0.9 and a diameter ratio ( $d / D$ ) of 0.4 , when the bubble tab was placed at an angle of $15^{\circ}$ to stagnation points' axis (X-axis) with the axis serving as their symmetry in the flow upstream. Figure 8 (a) shows the plot comparing the drag coefficient at the optimized position with that of the main cylinder (tab-less), while in Figure 8 (b) the coefficient of drag for different optimized tab position (design point) were considered for the twin tab cylinder. A drag coefficient of 0.4069 was attained around the main cylinder with twin tab at the optimum position such that there was about $71.35 \%$ reduction of drag when compared with the drag coefficient around the main cylinder only.
Effect of Spacing Ratio ( $x / D)$ : A slight increase in the drag coefficient was observed as the spacing ratio of the twin bubble tab was increased, except when the bubble tab was at the flow separation point where it decreased steadily with the spacing ratio. Figure 9 (a) presents the relationship between the spacing ratio and drag coefficient for twin bubble tabs at a constant diameter ratio (d/D) of 0.2 .

Effect of Diameter Ratio (d/D): Comparatively, in Figure 9 (b) the effect of the diameter ratio on the drag coefficient as it was being varied is indicated. From thence, it could be observed that the drag coefficient decreased as the diameter ratio increased except at the flow separation point where there is an increase in drag coefficient with increasing diameter ratio. This behavior is similar to that of the single bubble tab for both spacing ratio and diameter ratio. Likewise, it can be seen from the results that the flow separation was delayed due to flow interactions between the vortices generated by the bubble tabs and those generated by the main cylinder, thus reducing the quick formation of the Karman street, which implies a significant reduction of vortex shedding, but for the twin tab there is a delay in the formation of the upper vortices leading to the Karman street.

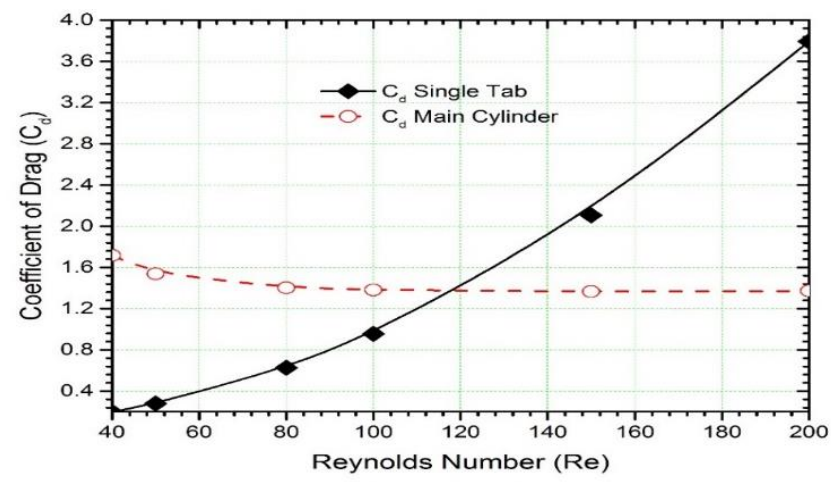

Figure 7. Graph Relating $C_{d}$ and Re (Single Bubble Tab) 


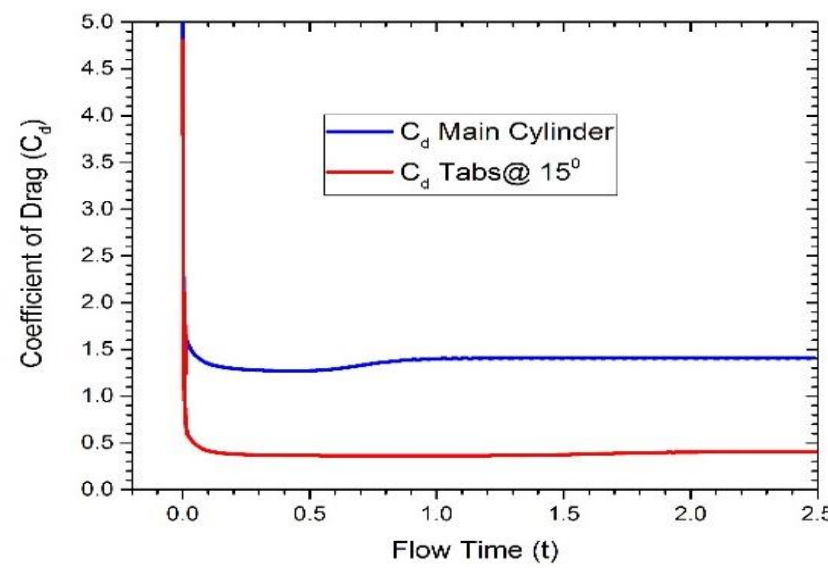

(a)

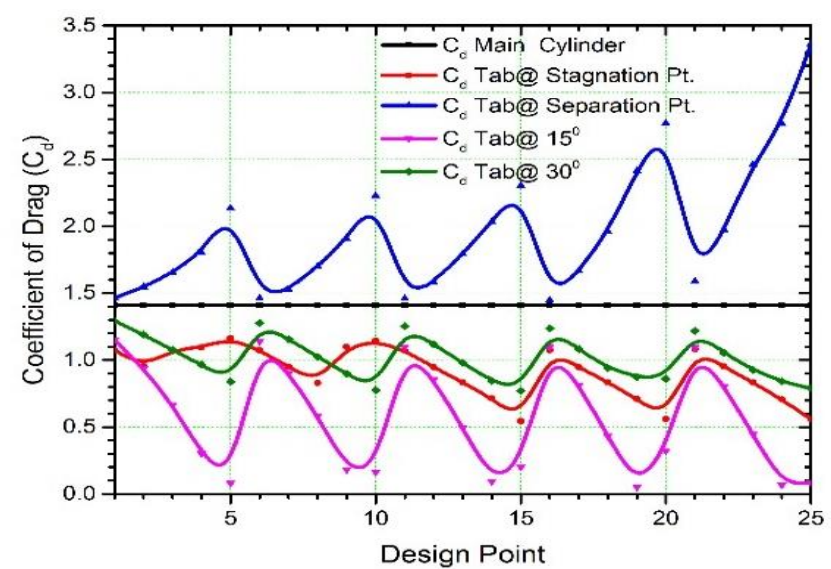

(b)

Figure 8: (a) Comparison of Cd Plot of Isolated Main Cylinder and Optimized Twin Bubble Tab at $15^{\circ}$ (b) Determination of Optimized position of the bubble (for twin tabs)

Figure 10 presents the vorticity contour of the twin tab configuration at $\mathrm{Re}=80$ and that of the main cylinder. It can be observed that the presence of the twin bubble tabs had lowered the flow velocity (and consequently, a reduction in momentum), just before the fluid reaches the main tab, thus resulting in the elongation of the vortex before breaking off from the prime vortex. The streamline of the flow is shown in Figure 11, where it was observed that the uniform pressure distribution formed around the isolated main cylinder was also reduced as a result of greater flow retardation around the main cylinder, a scenario that is in agreement with the potential flow theory. The pressure contour generated behaved similarly to that of the single bubble tab configuration, but with a less pressure distribution. The transformed pressure distribution is shown in Figure 12.

Effect of Reynolds Number at Optimized position: The Reynolds numbers' effect on the drag coefficient was also considered at the optimized position and it was observed that the drag reduced steadily with an increase in the Reynolds number. This is contrary to the result generated for the single bubble tab. This feature is captured in Figure 13 wherein, the relationship between the Reynolds number and the drag coefficient at the optimized tab position that compares the result generated with that of the main cylinder (tab-less) only, is presented.

\section{CONCLUSIONS AND RECOMMENDATION}

This research has presented the control of vortex shedding or VIV around a circular cylinder using a passive control (bubble tab) and on the basis of the simulation results generated, the following conclusions were drawn.

1. For the single bubble tab configuration, the peak drag reduction can be attained when the bubble tab is positioned at the stagnation point with a spacing ratio (x/D) of 1.2 and a diameter ratio (d/D) of 0.5 . The bubble should never be placed at the flow separation point as it tends to magnify the vortex shedding effect around the cylinder.

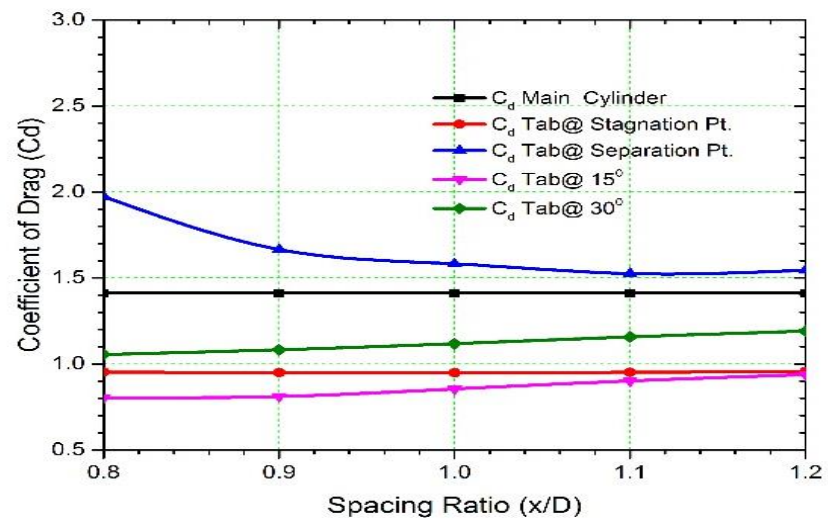

(a)

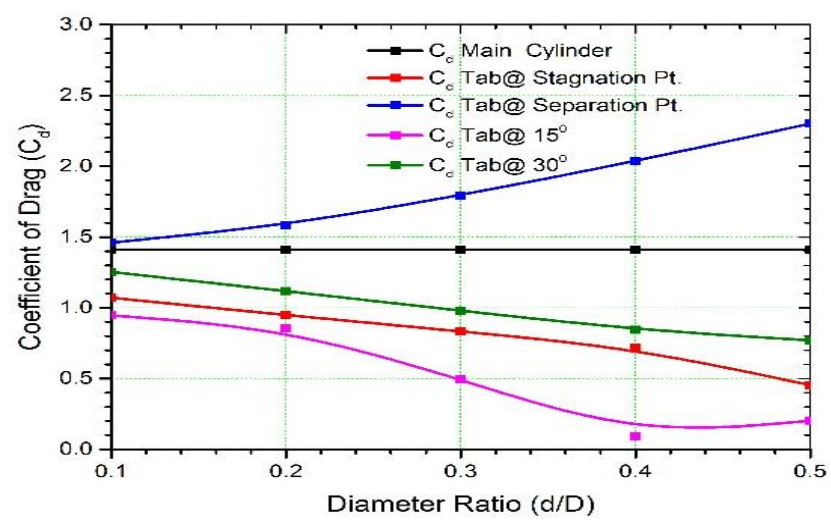

(b)

Figure 9: (a) Effect of spacing ratio on drag coefficient (at constant diameter ratio) (b) Effect of diameter ratio on drag coefficient (at constant spacing ratio)

Vol. 39, No. 4, October 2020 


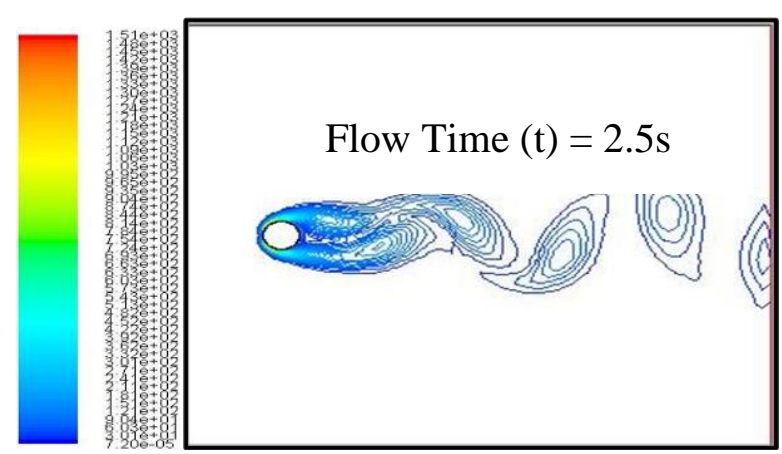

(a)

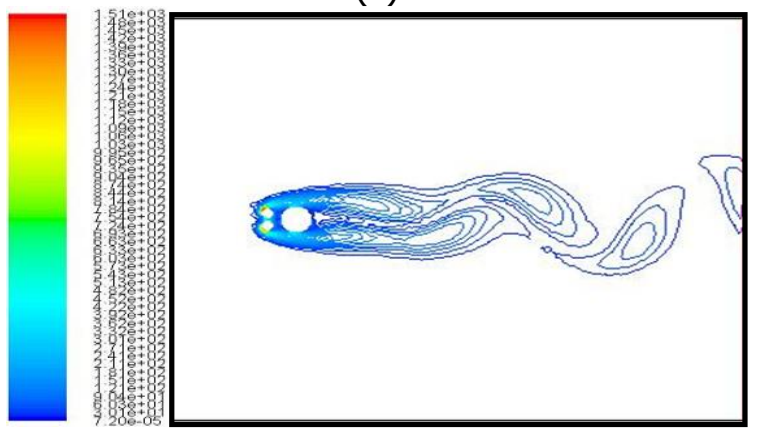

(b)

Figure 10 Vorticity Contour for the Main Cylinder only and Optimized Twin Bubble Tab, (a) The main cylinder only (b) the main cylinder with twin tab at

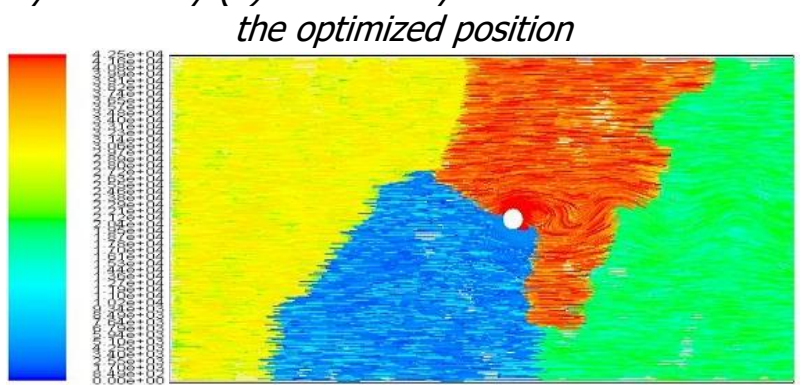

(a)

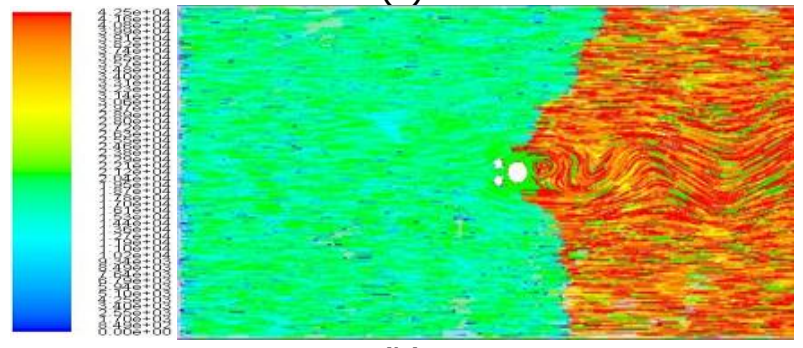

(b)

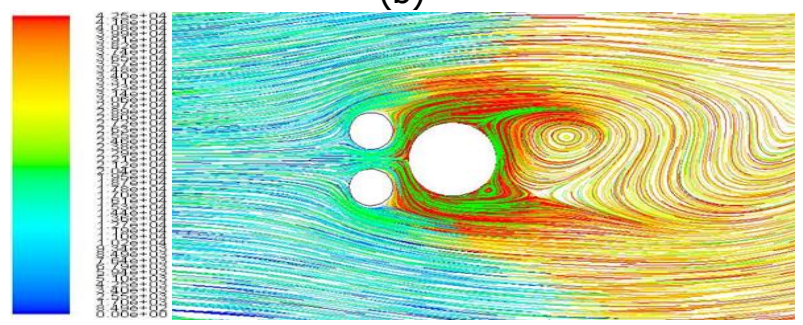

(c)

Figure 11: Streamline for Flow for isolated main cylinder and Optimized twin tab, (a) isolated main cylinder (b) twin tab at optimized position (c) zoomed ' $b$ '

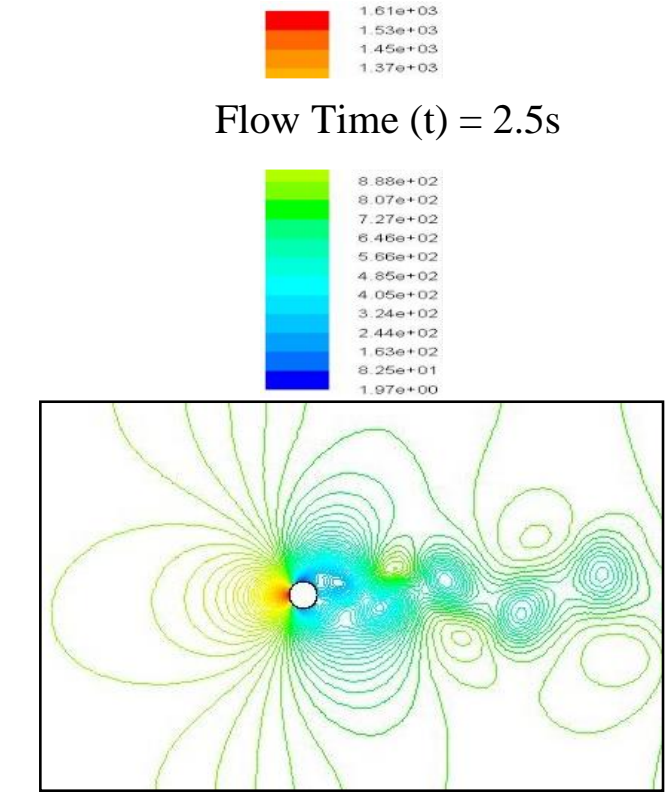

(a)

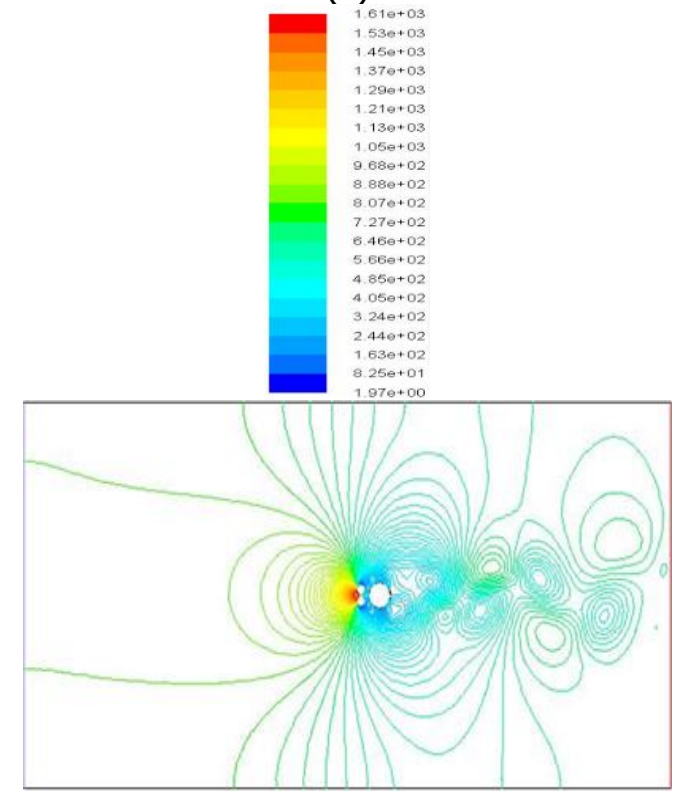

(b)

Figure 12: Pressure Contour at the Optimized Position (a) for isolated main cylinder (b) twin tab configuration

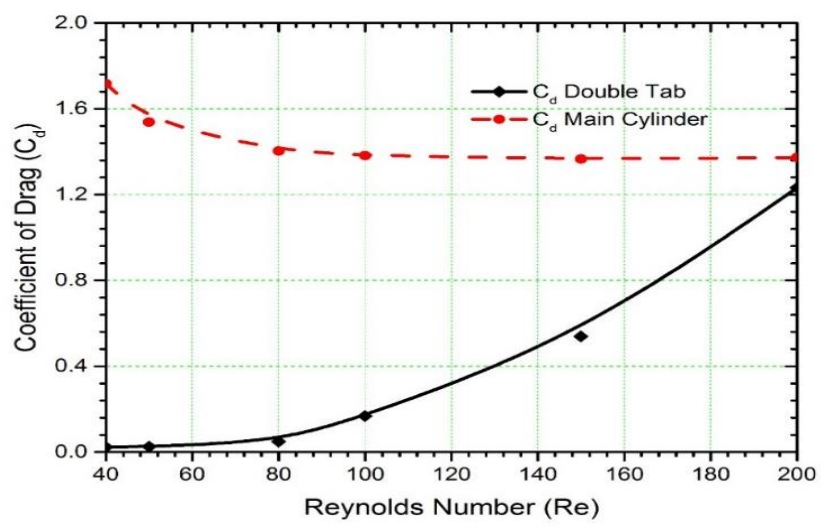

Figure 13: Graph Relating Cd and Re (Twin Bubble $T a b)$ 
2. For the twin bubble tab configuration, in order to achieve an optimal control of vortex shedding around the main cylinder using twin bubble tabs, the tabs must be positioned around the optimized position $\left(x / D=0.9 ; d / D=0.4 ; 15^{\circ}\right)$ and never at the flow separation point as it would rather be detrimental due to the amplification of vortex shedding around the main cylinder. The twin bubble tab was observed to control the formation of vortex better than the single bubble tab, indicating that the positioning of more tabs would afford a better control of vortex shedding at the optimized configuration.

3. In totality, the performance of the single and twin bubble tabs establishes that the drag coefficient which is a major factor contributing to vortex shedding around bluff bodies can be reduced without the need for an energy source unlike in the case of active controls.

Further study could be carried out considering more bubble tabs at different configurations to evaluate the effect on the drag and lift coefficients respectively.

\section{REFERENCES}

[1] M. Laroussi, M. Djebbi, and M. Moussa, "Triggering vortex shedding for flow past circular cylinder by acting on initial conditions A numerical study," Comput. Fluids, vol. 101, no. March 2019, pp. 194-207, 2014..

[2] J. D. Holmes, Wind loading on structures, Spon Press (Taylor and Francis Group), 2001.

[3] M. J. Every, R. King, and D. S. Weaver, "Vortexexcited vibrations of cylinders and cables and their suppression," Ocean Eng., vol. 9, no. 2, pp. 135-157, 1982.

[4] M. M. Zdravkovich, "Review and classification of various aerodynamic and hydrodynamic means for suppressing vortex shedding," J. Wind Eng. Ind. Aerodyn., vol. 7, pp. 145-189, 1981.

[5] J. Wu, C. Shu, and N. Zhao, "Investigation of flow characteristics around a stationary circular cylinder with an undulatory plate," Eur. J. Mech. B/Fluids, vol. 48, pp. 27-39, 2014.

[6] S. Mittal and a. Raghuvanshi, "Control of vortex shedding behind circular cylinder for flows at low Reynolds numbers," Int. J. Numer. Methods Fluids, vol. 35, no. December 1997, pp. 421447, 2001.

[7] L. Lu et al., "Numerical investigation of fluid flow past a circular cylinder with multiple control rods at low Reynolds number," J. Fluids
Struct., vol. 48, pp. 235-259, 2014.

[8] G. Nazeer, S. U. Islam, and S. H. Shigri, "Numerical investigation of vortex shedding mechanism for staggered rows of cylinders," Heliyon, vol. 5, no. 2, pp. 12-24, 2019.

[9] C. Williamson, "Sinusoidal flow relative to circular cylinders," J. Fluid Mech., vol. 155, pp. 141-174, 1985.

[10] M. T. Asyikin, "CFD Simulation of Vortex Induced Vibration of a Cylindrical Structure," Norwegian University of Science and Technology, June 2012.

[11] M. Sumer and J. Fredsoe, Hydrodynamics around cylindrical structures, Revised., vol. 26, no. 1. World Scientilfic Publishing Co. Plc. Ltd. https://doi.org/10.1016/s03783839(97)00031-8, 1997.

[12] K. Lam and L. Zou, "Three-dimensional numerical simulations of cross-flow around four cylinders in an in-line square configuration," $J$. Fluids Struct., vol. 26, no. 3, pp. 482-502, 2010.

[13] O. Posdziech and R. Grundmann, "A systematic approach to the numerical calculation of fundamental quantities of the two-dimensional flow over a circular cylinder," J. Fluids Struct., vol. 23, no. 3, pp. 479-499, 2007.

[14] H. Sucker, D.; Brauer, "Fluiddynamik Bei der Angestromten Zilindern.," Wärme Stoffubertragun, vol. 53, no. 9, pp. 1689-1699, 2013.

[15] E. Silva and A. L. Lima, "Numerical simulation of two-dimensional flows over a circular cylinder using the immersed boundary method," vol. 189, pp. 351-370, 2003.

[16] P. Badami, V. Shrivastava, V. Saravanan, N. Hiremath, and K. N. Seetharamu, "Numerical Analysis of Flow past Circular Cylinder with Triangular and Rectangular Wake Splitter," pp. 422-429, 2012.

[17] S. Mittal, V. Kumar, and A. Raghuvanshi, "Unsteady incompressible flows past two cylinders in tandem and staggered arrangements," Int. J. Numer. Methods Fluids, vol. 25, no. 11, pp. 1315-1344, 1997.

[18] M. N. Linnick and H. F. Fasel, "A high-order immersed interface method for simulating unsteady incompressible flows on irregular domains," J. Comput. Phys., vol. 204, no. 1, pp. 157-192, 2005.

[19] A. B. Harichandan and A. Roy, "Numerical investigation of low Reynolds number flow past two and three circular cylinders using unstructured grid CFR scheme," Int. J. Heat Fluid Flow, vol. 31, no. 2, pp. 154-171, 2010. 